\title{
Loss of CDH1 (E-cadherin) expression is associated with infiltrative tumour growth and lymph node metastasis
}

Sun A Kim ${ }^{1,10}$, Kentaro Inamura ${ }^{1,2,10}$, Mai Yamauchi ${ }^{1,10}$, Reiko Nishihara ${ }^{1,3,10}$, Kosuke Mima ${ }^{1,10}$, Yasutaka Sukawa ${ }^{1}$, Tingting $\mathrm{Li}^{1,4}$, Mika Yasunari ${ }^{1}$, Teppei Morikawa ${ }^{5}$, Kathryn C Fitzgerald ${ }^{6}$, Charles S Fuchs ${ }^{1,7}$, Kana Wu ${ }^{3}$, Andrew T Chan ${ }^{7,8}$, Xuehong Zhang ${ }^{7}$, Shuji Ogino ${ }^{\star}, 1,6,9,11$ and Zhi Rong Qian ${ }^{\star, 1,11}$

${ }^{1}$ Department of Medical Oncology, Dana-Farber Cancer Institute and Harvard Medical School, 450 Brookline Avenue M420, Boston, MA 02215, USA; ${ }^{2}$ Laboratory of Human Carcinogenesis, National Cancer Institute, National Institutes of Health, 9000 Rockville Pike, Bethesda, MD 20892, USA; ${ }^{3}$ Department of Nutrition, Harvard T.H. Chan School of Public Health, 655 Huntington Avenue Building 2, room 213, Boston, MA 02115, USA; ${ }^{4}$ Department of Geriatric Gastroenterology, Chinese PLA General Hospital, 28 Fuxing Road, Haidian, Beijing 100853, China; ${ }^{5}$ Department of Pathology, The University of Tokyo Hospital, 7-3-1 Hongo, Bunkyo-ku, Tokyo 113-0033, Japan; ${ }^{6}$ Department of Epidemiology, Harvard T.H. Chan School of Public Health, 655 Huntington Avenue, Boston, MA 02115, USA; ${ }^{7}$ Channing Division of Network Medicine, Department of Medicine, Brigham and Women's Hospital, and Harvard Medical School, 29 Wigglesworth Street, Boston, MA 02120, USA; ${ }^{8}$ Division of Gastroenterology, Massachusetts General Hospital and Harvard Medical School, 55 Fruit Street, GRJ-722, Boston, MA 02114, USA and 'Department of Pathology, Brigham and Women's Hospital, and Harvard Medical School, 29 Wigglesworth Street, Boston, MA 02120, USA

Background: Loss of CDH1 (E-cadherin) expression in cancer cells may promote cell migration and invasion. Therefore, we hypothesised that loss of $\mathrm{CDH} 1$ expression in colorectal carcinoma might be associated with aggressive features and clinical outcome.

Methods: Utilising molecular pathological epidemiology database of 689 rectal and colon cancer cases in the Nurses' Health Study and the Health Professionals Follow-up Study, we assessed tumour CDH1 expression by immunohistochemistry. Multivariate logistic regression analysis was conducted to assess association of CDH1 loss with tumour growth pattern (expansileintermediate vs infiltrative) and lymph node metastasis and distant metastasis, controlling for potential confounders including microsatellite instability, CpG island methylator phenotype, LINE-1 methylation, and PIK3CA, BRAF and KRAS mutations. Mortality according to $\mathrm{CDH} 1$ status was assessed using Cox proportional hazards model.

Results: Loss of tumour CDH1 expression was observed in 356 cases (52\%), and associated with infiltrative tumour growth pattern (odds ratio (OR), 2.02; 95\% confidence interval $(\mathrm{Cl}), 1.23-3.34 ; P=0.006)$ and higher $\mathrm{pN}$ stage $(\mathrm{OR}, 1.73 ; 95 \% \mathrm{Cl}$, 1.23-2.43; $P=0.001)$. Tumour $\mathrm{CDH} 1$ expression was not significantly associated with distant metastasis or prognosis.

Conclusions: Loss of $\mathrm{CDH} 1$ expression in colorectal cancer is associated with infiltrative tumour growth pattern and lymph node metastasis.

\footnotetext{
*Correspondence: Dr S Ogino; E-mail: shuji_ogino@dfci.harvard.edu or Dr ZR Qian; E-mail: zhirong_qian@dfci.harvard.edu

${ }^{10}$ These authors contributed equally as co-first authors.

${ }^{11}$ These authors contributed equally as co-last authors.
}

Received 13 February 2015; revised 6 August 2015; accepted 4 September 2015; published online 7 January 2016

(c) 2016 Cancer Research UK. All rights reserved 0007-0920/16 
Infiltrative growth pattern at the tumour margin is associated with shorter patient survival in colorectal cancer (Cianchi et al, 2007; Karamitopoulou et al, 2015; Keum et al, 2012; Morikawa et al, 2012; Zlobec et al, 2009, 2007). Infiltrative growth pattern has been associated with specific tumour subtype; that is, microsatellitestable (MSS) and BRAF-mutated colorectal cancer (Morikawa et al, 2012; Roman et al, 2010). However, the mechanism underlying the tumour infiltration remains uncertain.

CDH1 (the HUGO-approved official symbol for cadherin-1; HGNC ID: 1748; aka E-cadherin) is a calcium-dependent transmembrane protein that facilitates the assembly of specialised intercellular junctions necessary for the adherence of epithelial cells (Qian et al, 2007; Tsanou et al, 2008). Several in vitro experiments have shown that downregulation of $\mathrm{CDH} 1$ expression in colon cancer cells promotes cell migration and invasion (Chen et al, 2012; Lu et al, 2012). However, the association of tumour CDH1 expression in human colorectal cancer tissue with aggressive tumour behaviour remains controversial. Some studies have shown that loss of CDH1 expression is associated with lymph node metastasis (Karamitopoulou et al, 2011; Kwak et al, 2007; Lugli et al, 2007), distant metastasis (Jie et al, 2013) and higher mortality (Filiz et al, 2010; Jie et al, 2013; Lugli et al, 2007). In contrast, other studies have shown no association of loss of CDH1 expression with lymph node metastasis (Filiz et al, 2010), distant metastasis (Filiz et al, 2010) or higher mortality (Bondi et al, 2006; Kwak et al, 2007; Zlobec et al, 2007). We hypothesised that loss of tumour $\mathrm{CDH} 1$ expression might be associated with infiltrative tumour growth pattern and metastasis of colorectal cancer. We could evaluate the association of $\mathrm{CDH} 1$ status, controlling for potential confounders such as $\mathrm{CpG}$ island methylator phenotype (CIMP), MSI, long interspersed nucleotide element-1 (LINE-1) hypomethylation, and PIK3CA, BRAF and KRAS mutations.

\section{MATERIALS AND METHODS}

Study population and ascertainment of mortality. We used a database from two prospective cohort studies, the Nurses' Health Study (NHS, $N=121701$ women observed since 1976) and the Health Professionals Follow-up Study (HPFS, N=51529 men observed since 1986) (Morikawa et al, 2012). Every 2 years, participants were sent follow-up questionnaires to collect information on health and lifestyle factors, and asked whether they had received diagnoses of major diseases including cancer. Follow-up has exceeded $90 \%$ for each 2 -year questionnaire. The National Death Index was used to ascertain deaths of study participants and identify unreported lethal colorectal cancer cases. For nearly all incident colorectal cancer cases, medical records were reviewed. If a patient was deceased, the cause of death was assigned by study physicians. Disease stage was evaluated based on American Joint Committee on Cancer (AJCC) stage of colorectal cancer. Formalinfixed paraffin-embedded tissue blocks were collected from hospitals where participants with colorectal cancer had undergone tumour resection. Based on the availability of adequate specimens for pathological evaluation, tumour $\mathrm{CDH} 1$ expression data, and survival data, 689 stage I to IV colorectal cancer cases of diagnosed up to 2008 were included in this study. This study represents a new analysis of $\mathrm{CDH} 1$ expression in colorectal cancer, combined with pre-existing data of microsatellite instability (MSI), MLH1 promoter methylation, CIMP, LINE-1 hypomethylation, and $B R A F, K R A S$ and PIK3CA mutations in our database. Patients were observed until death, or January 2012, whichever came first. Written informed consent was obtained from all study participants. This study was approved by the Human Subjects
Committees at Harvard T.H. Chan School of Public Health and Brigham and Women's Hospital.

Histopathologic evaluations. Haematoxylin and eosin-stained tissue sections from all colorectal cancer cases were reviewed by a single pathologist (SO) unaware of other clinical or molecular data. Tumour grade was categorised as high $(\leqslant 50 \%$ glandular area) or low ( $>50 \%$ glandular area). Tumour growth pattern at the tumour margin was evaluated at low-power magnification and categorised as expansile, intermediate or infiltrative (Figure 1) as previously described (Morikawa et al, 2012). The growth pattern was considered expansile when tumour margin was pushing and reasonably well-circumscribed. It was considered intermediate when tumour border was blurred by invasion of large or mediumsized tumour glands. It was considered infiltrative when small tumour glands or irregular clusters or cords of tumour cells invaded in a diffuse manner with widespread penetration of normal tissue without distinct border. Tumours with a small microscopic focus of an infiltrative growth pattern were considered intermediate. A subset of cases $(>100)$ was reviewed by a second pathologist (TM). Concordance between the two pathologists was as follows (all $P<0.001$ ): 0.77 (weighted $\kappa=0.62$ ) for trichotomised tumour growth pattern (expansile, intermediate and infiltrative); 0.96 (weighted $\kappa=0.73$ ) for dichotomised tumour growth pattern (expansile-intermediate $v s$ infiltrative); and 0.96 (weighted $\kappa=0.72$ ) for tumour grade (low $v s$ high).

Immunohistochemistry. Tissue microarrays (TMAs) were constructed as previously described (Ogino et al, 2006b). Haematoxylin and eosin-stained slides were reviewed by a pathologist (SO) to mark highly cellular portions. Two $0.6 \mathrm{~mm}$ tissue cores each from tumour and normal colonic mucosa were placed in each TMA block. TMAs were constructed using Automated Arrayer (Beecher Instruments, Sun Prairie, WI, USA). Expression of CDH1 was determined by immunohistochemical staining. The TMA slides were deparaffinised with xylene overnight and consecutively with $100 \%$ alcohol for $15 \mathrm{~min}$. For antigen retrieval, the slides were merged in citrate buffer at $\mathrm{pH} 9.0$ (pH 9.0, BioGenex, Fremont, CA, USA) and were microwaved in pressure cooker for $5 \mathrm{~min}$ at $100{ }^{\circ} \mathrm{C}$. After cooling at room temperature for $45 \mathrm{~min}$ and rinsing with tris-buffered saline (TBS), the slides were incubated with $10 \%$ normal goat serum (Vector Laboratories, Burlingame, CA) in phosphate-buffered saline for $30 \mathrm{~min}$. The slides were incubated with $\mathrm{CDH} 1$ primary antibody (mouse monoclonal, 1:75 dilution, Dako, Carpinteria, CA, USA) for $40 \mathrm{~min}$. After warming to room temperature and thorough washing with TBS, we applied a secondary biotinylated rabbit anti-mouse immunoglobulin (Dako) for $30 \mathrm{~min}$. After washing sections in TBS, the signal was detected by the avidin-biotin complex system and diaminobenzidine tetrahydrochloride (Dako) for $5 \mathrm{~min}$ for colour development, washed with distilled water and counterstained with haematoxylin. A positive signal was indicated by a reddish-brown stain.

Tumour CDH1 expression was categorised into intact or lost (Figure 2). Intact CDH1 expression was defined when most of the tumour cells were recognisably stained with $\mathrm{CDH} 1$ in complete circumference of the membrane. No staining or faint staining in part of total tumour cell membrane was considered loss of CDH1 expression (Fadare et al, 2005; He et al, 2013; Toth et al, 2012). Methods of immunohistochemical staining and interpretations for CTNNB1 have been described previously (Morikawa et al, 2011).

All immunostained slides for each marker were interpreted by a pathologist (CDH1 by SAK and CTNNB1 by TM) unaware of other data. A subset of $>100$ cases (for each marker) was reviewed independently by a second pathologist (CDH1 by KI and CTNNB1 by $\mathrm{SO}$ ) unaware of other data. The Spearman correlation coefficient between the two observers was 0.72 for CDH1 (weighted $\kappa=0.72, P<0.001$ ), and 0.86 for membrane CTNNB1 (weighted $\kappa=0.72, P<0.0001$ ) indicating substantial agreement. 

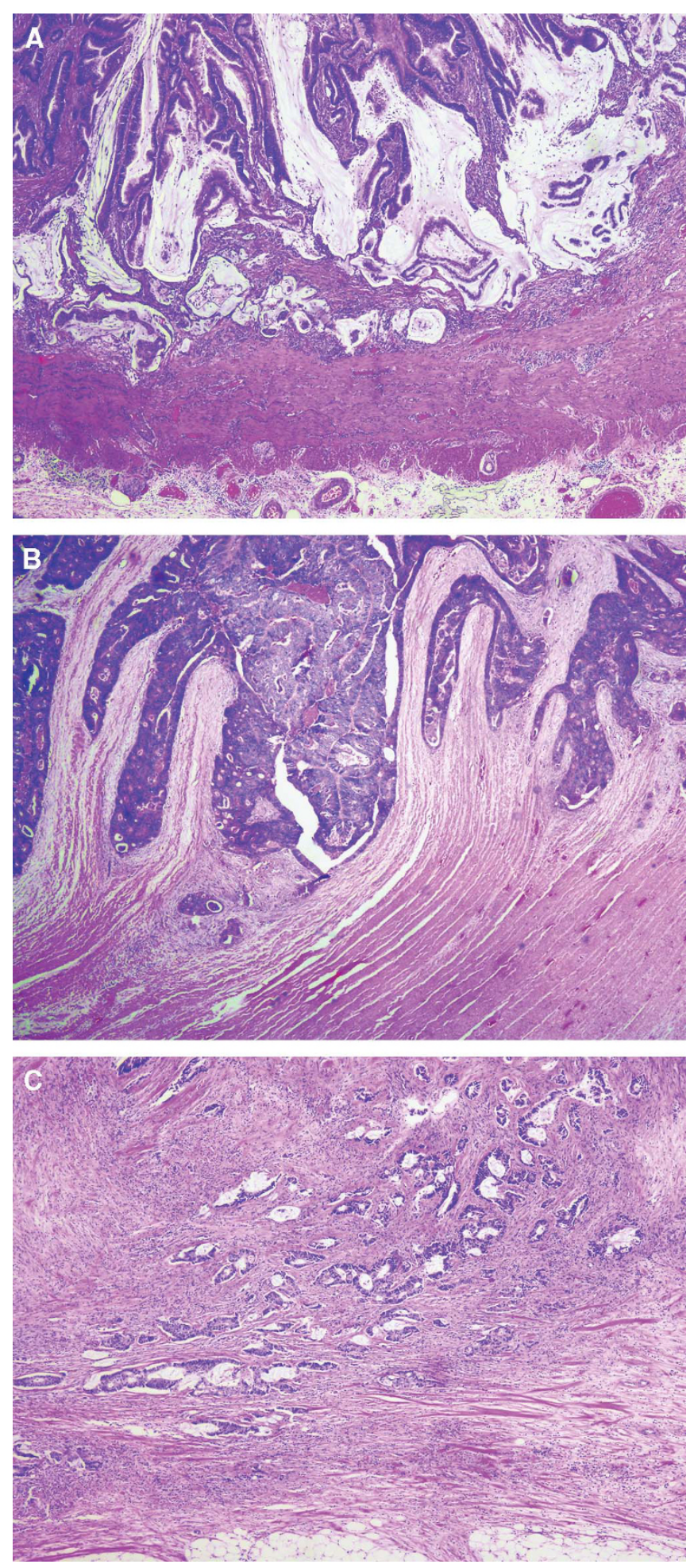

Figure 1. Tumour growth pattern. (A) Expansile growth pattern is defined by pushing and well-circumscribed tumour border.

(B) Intermediate growth pattern is defined by tumour border blurred by invasion of large or medium-sized tumour glands. (C) Infiltrative growth pattern is designated when small tumour glands or irregular clusters or cords of tumour cells infiltrate in a diffuse manner without distinct border. Haematoxylin and eosin stain, original magnification $\times 40$.

MSI analysis. All tumour molecular analyses were carried out completely blinded to patient identity, clinical features and outcome data (Morikawa et al, 2012). DNA was extracted from archival paraffin-embedded colorectal carcinoma tissue
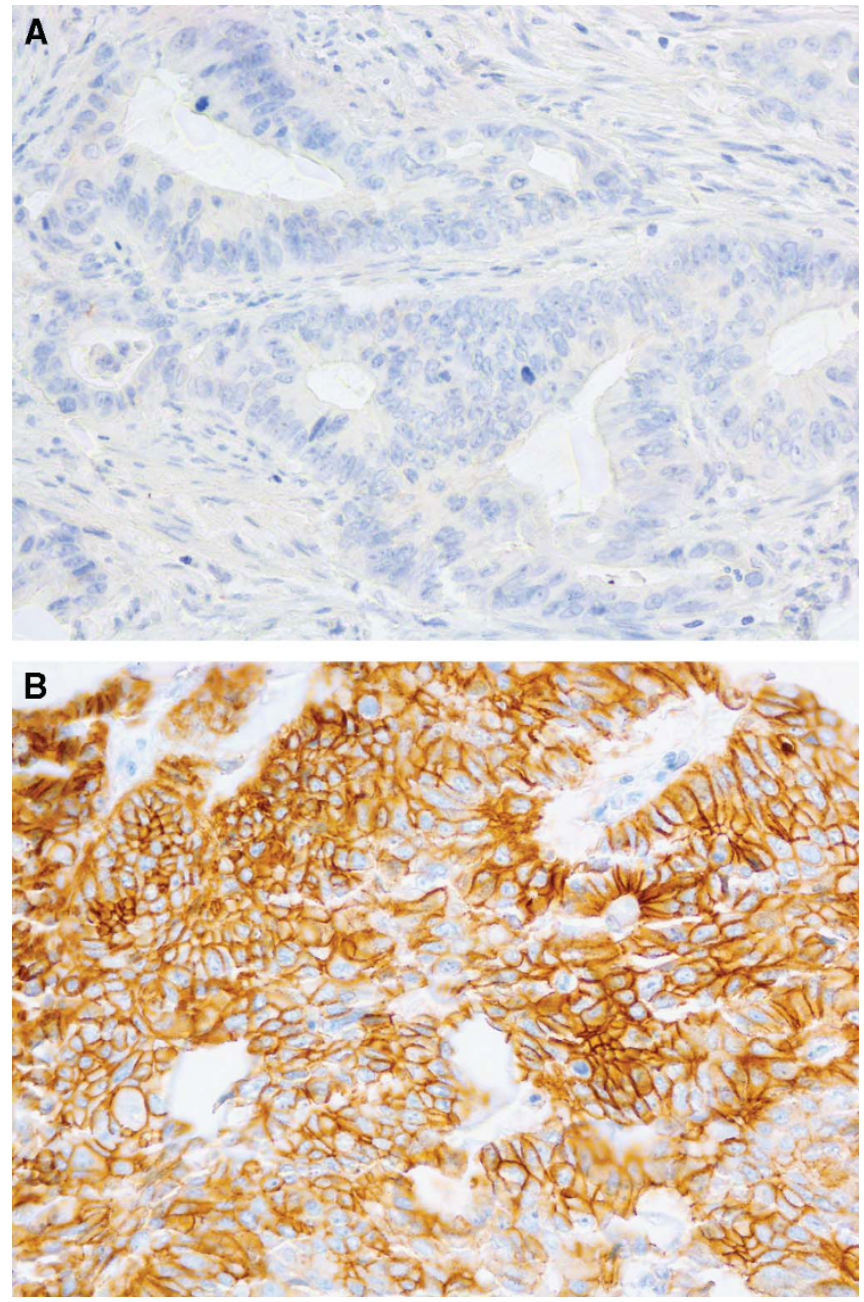

Figure 2. $\mathrm{CDH} 1$ immunohistochemistry. (A) Lack of staining in tumour cell membrane is interpreted as loss of $\mathrm{CDH} 1$ expression. (B) Brown staining in complete circumference of tumour cell membrane is interpreted as intact $\mathrm{CDH} 1$ expression. Original magnification $\times 400$.

(Ogino et al, 2006b, 2005a). We marked tumour areas on haematoxylin and eosin-stained slides, and dissected tumour tissue by a sterile needle. MSI analysis was carried out by PCR using a panel of 10 microsatellite markers (D2S123, D5S346, D17S250, BAT25, BAT26, BAT40, D18S55, D18S56, D18S67 and D18S487) as previously described (Ogino et al, 2006a, 2009). MSI-high was defined as the presence of instability in $\geqslant 30 \%$ of the markers and microsatellite stable (MSS) as no instability of all markers. The tumours with MSI-low, defined as the presence of instability in 10$29 \%$ of the markers, showed similar features with MSS tumours (Ogino et al, 2006a, 2009). Therefore, we combined MSI-low and MSS tumours into one group.

Methylation analyses for CpG islands and LINE-1. We quantified DNA methylation in eight CIMP-specific promoters (CACNA1G, CDKN2A, CRABP1, IGF2, MLH1, NEUROG1, RUNX3 and SOCS1) (Hinoue et al, 2012; Nosho et al, 2008; Ogino et al, 2008). Bisulphite treatment on genomic DNA and subsequent real-time PCR (MethyLight) were performed as previously described (Ogino et al, 2006c, 2009). CIMP-high was defined as the presence of six or more methylated promoters, CIMP-low as one to five methylated promoters and CIMP-negative as the absence (zero out of eight) of methylated promoters, according to the previously established criteria (Hinoue et al, 2012; Ogino et al, 2007, 2009). Methylation levels of LINE-1 repetitive elements were quantified by validated 
bisulphite DNA treatment, PCR and pyrosequencing assay as previously described (Irahara et al, 2010; Ogino et al, 2006c, 2008).

Sequencing of BRAF, KRAS and PIK3CA. With DNA extracted from archival paraffin-embedded colon cancer tissue, PCR and pyrosequencing covering BRAF (codon 600) (Ogino et al, 2005b), KRAS (codons 12, 13, 61 and 146) (Imamura et al, 2014; Ogino et al, 2005a) and PIK3CA (exons 9 and 20) (Morikawa et al, 2012) were performed.

Statistical analysis. All statistical analyses were conducted using SAS software (version 9.3; SAS Institute Inc., Cary, NC, USA). All $P$-values were two sided. To assess associations between categorical data, the $\chi^{2}$-test was performed. To compare mean age and mean LINE-1 methylation levels, Student's $t$-test was performed. All cross-sectional univariable analyses for clinical, pathological and tumour molecular associations were secondary analyses, and we adjusted two-sided $\alpha$ level to $0.003(=0.05 / 17)$ by simple Bonferroni correction for multiple hypothesis testing.

The association of CDH1 expression with tumour growth pattern, lymph node metastasis or distant metastasis was assessed by multivariate logistic regression analyses, controlling for covariates including sex, age (continuous, increase by 10 years), year of diagnosis (continuous, increase by 1 year), family history of colorectal cancer in first degree relatives (absent vs present), tumour location (caecum $v s$ ascending to transverse colon $v s$ splenic flexure to sigmoid colon vs rectum), MSI (low/MSS vs high), CIMP (negative/low vs high), LINE-1 hypomethylation (10\% decrease, continuous), BRAF (mutant vs wild type), KRAS (mutant $v s$ wild type), PIK3CA (mutant vs wild type) and CTNNB1 membrane expression (intact $v s$ lost). A backward stepwise elimination with a threshold of $P=0.10$ was used to select variables in the final model to avoid overfitting. Odds ratios (OR) were adjusted for missing values of all variables in the final model.

For survival analyses, we used multivariate Cox proportional hazards regression models to compute mortality hazard ratio (HR), controlling for potential confounders. The covariates included in the initial models were sex, age (continuous, increase by 10 years), year of diagnosis (continuous, increase by 1 year), family history of colorectal cancer in first degree relatives (absent $v s$ present), tumour location (caecum $v s$ ascending to transverse colon $v s$ splenic flexure to sigmoid colon $v s$ rectum), tumour grade (low $v s$ high), tumour growth pattern (expansile-infiltrative $v s$ infiltrative), MSI (low/MSS vs high), CIMP (negative/low vs high), LINE-1 hypomethylation ( $10 \%$ decrease, continuous), BRAF (mutant vs wild type), KRAS (mutant vs wild type), PIK3CA (mutant vs wild type) and CTNNB1 membrane expression (intact vs lost). A backward stepwise elimination was performed with a threshold of $P=0.05$ to avoid overfitting. AJCC colorectal cancer stage (I, II, III, IV and missing) was used as a stratifying variable using the strata option in the SAS 'proc phreg' command to avoid overfitting and residual confounding. We incorporated cases with missing information into the majority category of the given covariate. We confirmed that excluding cases with missing information in any of the covariates did not substantially alter results (data not shown). Proportion of missing values for each variable was as follows: sex, $0 \%$; age, $0 \%$; year of diagnosis, $0 \%$; family history of colorectal cancer in first degree relatives, $0.4 \%$; body mass index, $0.1 \%$; tumour location, $0.3 \%$; tumour grade $0.3 \%$, MSI status, 2.4\%; CIMP status, 7.1\%; LINE-1 methylation level, 2.6\%; BRAF mutation, $1.9 \%$; KRAS mutation, $1.7 \%$; and PIK3CA mutation, 7.6\%; CTNNB1 membrane expression, $4.4 \%$. The proportionality of hazards assumption was satisfied by evaluating a time-dependent variable, which was the cross-product of CDH1 expression variable and survival time $(P>0.20)$.

\section{RESULTS}

CDH1 expression in colorectal cancer. Among the 689 colorectal cancer cases, 97 cases (15\%) metastasised to distant sites. There were 389 cases $(62 \%)$ without lymph node metastasis (AJCC N0), $151(24 \%)$ with metastasis in 1 to 3 lymph nodes (AJCC N1) and 88 (14\%) with metastasis in $\geqslant 4$ more lymph nodes (AJCC N2). CDH1 expression was preserved in $333(48 \%)$ and lost in 356 (52\%). Table 1 summarises clinical, pathological and molecular features according to $\mathrm{CDH} 1$ expression. Loss of CDH1 expression was associated with older age, infiltrative tumour growth pattern and BRAF mutation $(P \leqslant 0.003$ with the adjusted $\alpha$ level of 0.003 for multiple hypothesis testing).

Association of $\mathrm{CDH} 1$ expression with infiltrative tumour growth pattern and colorectal cancer metastasis. In multivariate logistic regression analyses, loss of $\mathrm{CDH} 1$ expression was significantly associated with infiltrative tumour growth pattern (OR, 2.02; 95\% CI, 1.23-3.34; $P=0.006$, Table 2), and with higher AJCC pN stage (OR, 1.73; 95\% CI, 1.23-2.43; $P=0.001$, Table 3), independent of other clinicopathologic and molecular features of colorectal cancer. We also assessed 317 cases that were positive for $\mathrm{CDH} 1$ in normal colorectal epithelial mucosa, and observed a similar association of loss of tumour $\mathrm{CDH} 1$ expression with the infiltrative tumour growth pattern, although statistical power was limited (Supplementary Table 1).

We also used the multivariable logistic regression model for the infiltrative tumour growth pattern that included combined MSI/BRAF status. In this exploratory analysis, in addition to loss of $\mathrm{CDH} 1$ expression, MSS/BRAF-mutant tumours were associated with the infiltrative tumour growth pattern (Supplementary Table 2).

Loss of CDH1 expression was not associated with distant metastasis in either univariate $(\mathrm{OR}, 1.18$; 95\% CI, 0.76-1.82; $P=0.46)$ and multivariate logistic regression analyses (OR, 1.14; 95\% CI, 0.72-1.83; $P=0.58)$.

CDH1 expression and colorectal cancer mortality. During a median follow-up of 14.3 months for 306 patents who were censored (interquartile range, 11.1-18.0 months), 383 patients died out of the 689 patients. Among 383 deaths, 208 deaths were colorectal cancer specific. Loss of CDH1 expression was not significantly associated with colorectal cancer-specific mortality or overall mortality (Table 4).

\section{DISCUSSION}

We tested the hypothesis that loss of $\mathrm{CDH} 1$ expression in colorectal carcinoma cells might be associated with tumour growth pattern, and metastasis to lymph nodes and distant sites. Loss of $\mathrm{CDH} 1$ expression was significantly associated with infiltrative tumour growth pattern and higher AJCC $\mathrm{pN}$ stage, but not metastasis to distant sites. These associations were independent of other clinical, pathological and molecular features of colorectal cancer. Our results implicate that tumour CDH1 expression may serve as a predictive marker for tumour invasion and lymph node metastasis.

Colorectal cancer is a heterogeneous group of cancers with various histological and molecular phenotypes that differ in disease progression (Morikawa et al, 2011). Infiltrative growth pattern at the tumour margin and lymph node metastasis are reliable histological indicators of higher colorectal cancer mortality (Cianchi et al, 2007; Keum et al, 2012; Morikawa et al, 2012; Zlobec et al, 2009, 2014, 2007). Considering that the effects of adjuvant therapy may differ according to tumour molecular features (Jonker et al, 2014; Liao et al, 2012), identifying tumour 
Table 1. Clinical, pathological and molecular characteristics in colorectal cancer cases according to tumour CDH1 expression

CDH1 expression

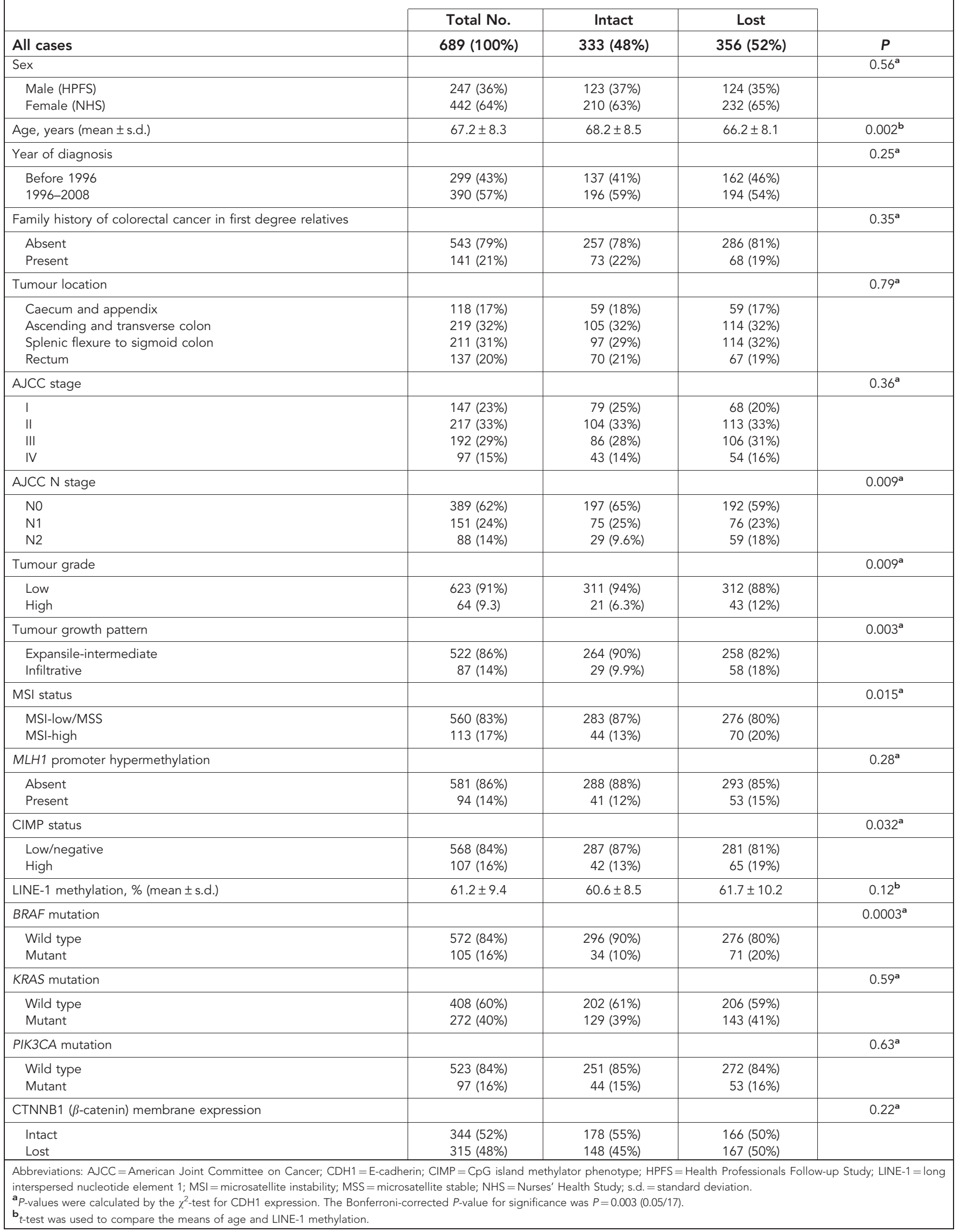


infiltration and metastasis-associated molecular markers is important for targeted therapy.

$\mathrm{CDH} 1$ is mainly responsible for adherence junctions between epithelial cells (Tsanou et al, 2008) and therefore implicated in the

\begin{tabular}{|c|c|c|}
\hline \multicolumn{3}{|c|}{$\begin{array}{l}\text { Table 2. Multivariate logistic regression analysis to assess } \\
\text { association between infiltrative tumour growth pattern and } \\
\text { loss of CDH1 expression }\end{array}$} \\
\hline $\begin{array}{l}\text { Predictors for infiltrative tumour growth } \\
\text { pattern (vs expansile-intermediate } \\
\text { growth pattern) }\end{array}$ & & $P$ \\
\hline Loss of CDH1 expression (vs intact) & $2.02(1.23-3.34)$ & 0.006 \\
\hline \multicolumn{3}{|l|}{ Covariates $^{a}$} \\
\hline $\begin{array}{l}\text { MSI-high (vs MSI-low/MSS) } \\
\text { BRAF mutation ( } v \text { s wild type) } \\
\text { Loss of CTNNB1 membrane expression ( } v s \text { intact) }\end{array}$ & $\begin{array}{l}0.11(0.04-0.31) \\
4.44(2.28-8.66) \\
1.62(0.98-2.68)\end{array}$ & $\begin{aligned}<0.0001 \\
<0.0001 \\
0.06\end{aligned}$ \\
\hline \multicolumn{3}{|c|}{$\begin{array}{l}\text { Abbreviations: } \mathrm{Cl}=\text { confidence interval; } \mathrm{CIMP}=\mathrm{CpG} \text { island methylator phenotype; } \\
\mathrm{LINE}-1=\text { long interspersed nucleotide element } 1 ; \mathrm{MSI}=\text { microsatellite instability; } \\
\mathrm{MSS}=\text { microsatellite stable; } \mathrm{OR}=\text { odds ratio. } \\
{ }^{\mathrm{a}} \mathrm{C} \text {; } \\
10 \text { yeariates included in the initial models were as follow: sex, age (continuous, increase by } \\
\text { history of colorectal cancer in first degree relatives (absent vs present), tumour location } \\
\text { (caecum, ascending to transverse colon, splenic flexure to sigmoid colon, rectum), MSI (low/ } \\
\text { MSS vs high), CIMP (negative/low vs high), LINE- } 1 \text { methylation ( } 10 \% \text { decrease, continuous), } \\
\text { BRAF, KRAS, PIK3CA mutations and CTNNB1 membrane expression (intact vs lost). } \\
\text { A backward stepwise elimination with a threshold of } P=0.10 \text { was used to select variables in } \\
\text { the final models. }\end{array}$} \\
\hline
\end{tabular}

Table 3. Multivariate logistic regression analysis to assess association between lymph node metastasis and loss of CDH1 expression

\begin{tabular}{|c|c|c|}
\hline $\begin{array}{l}\text { Predictors for higher AJCC N stage } \\
\text { (from } 0 \text { to } 2 \text {, ordinal categorical) }\end{array}$ & $\begin{array}{l}\text { Multivariate OR } \\
\qquad(95 \% \mathrm{Cl})\end{array}$ & $P$ \\
\hline Loss of CDH1 expression (vs intact) & $1.73(1.23-2.43)$ & 0.001 \\
\hline \multicolumn{3}{|l|}{ Covariates $^{a}$} \\
\hline $\begin{array}{l}\text { Family history of colorectal cancer in first } \\
\text { degree relatives } \\
\text { MSI-high (vs MSI-low/MSS) } \\
\text { LINE-1 methylation, } 10 \% \text { decrease }\end{array}$ & $\begin{array}{l}0.26(0.13-0.51) \\
1.25(1.04-1.51)\end{array}$ & $\begin{array}{c}<0.0001 \\
0.017\end{array}$ \\
\hline \multicolumn{3}{|c|}{ 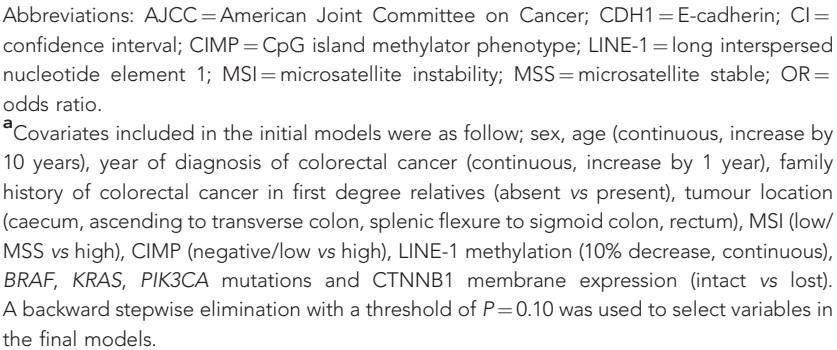 } \\
\hline
\end{tabular}

progression of tumour invasion (Chen et al, 2012; Lu et al, 2012). The role of $\mathrm{CDH} 1$ downregulation in colorectal cancer invasion is evidenced by several in vitro studies (Chen et al, 2012; Lu et al, 2012). However, in the histological level, the association of CDH1 loss with infiltrative growth pattern of colorectal cancer is not fully understood. One study of colorectal cancer (Zlobec et al, 2007) has identified $\mathrm{CDH} 1$ as a marker for tumour budding at the invasive margin in colorectal cancer. The study by Zlobec et al used a large number of cases $(N=1164)$, but all the cases were mismatch repair proficient (MSS) colorectal cancers (Zlobec et al, 2007). Our present results not only complement but also enhance previous data (Zlobec et al, 2007), after controlling for major clinical, pathologic and molecular characteristics of colorectal cancer.

In our study, loss of CDH1 expression was associated with higher $\mathrm{pN}$ stage, although it was not significantly associated with distant metastasis or colorectal cancer-specific mortality. A number of studies have investigated the association of loss of CDH1 expression with lymph node metastasis (Filiz et al, 2010; He et al, 2013; Karamitopoulou et al, 2011; Kwak et al, 2007; Lugli et al, 2007), distant metastasis (Filiz et al, 2010; He et al, 2013; Jie et al, 2013) or colorectal cancer mortality (Bondi et al, 2006; Filiz et al, 2010; He et al, 2013; Jie et al, 2013; Kwak et al, 2007; Lugli et al, 2007; Zlobec et al, 2007), but the results are conflicting. Notably, none of the previous studies have comprehensively examined the association of $\mathrm{CDH} 1$ expression with these aggressive tumour behaviours, together with major molecular features of colorectal cancer in a large number of cases. CIMP, MSI, LINE-1 methylation or BRAF mutation has been associated with patient survival in colorectal cancer (Lochhead et al, 2013; Ogino et al, 2008, 2009). Therefore, our current study is of particular importance because we evaluated association of tumour $\mathrm{CDH} 1$ expression to lymph node metastasis, distant metastasis and colorectal cancer-specific survival, controlling for major molecular signatures of colorectal cancer in two large prospective cohorts. Moreover, none of the previous studies has used incident cases within prospective cohort studies; instead, all of the previous studies used a convenience sample with unknown degrees of selection bias. Therefore, those previous studies cannot exclude a possibility that their findings might have been influenced by selection bias or confounding, though none of those papers have appropriately discussed this substantial weakness.

This study possesses several key advantages. Our study participants were distributed throughout the United States, and thus colorectal cancer cases from our cohort studies were more representative of cases in general population than those from several academic hospitals. Our rich tumour database enabled us to simultaneously assess tumour pathologic and molecular features to control for confounders. None of the previous studies on tumour

Table 4. Colorectal cancer mortality by CDH1 expression

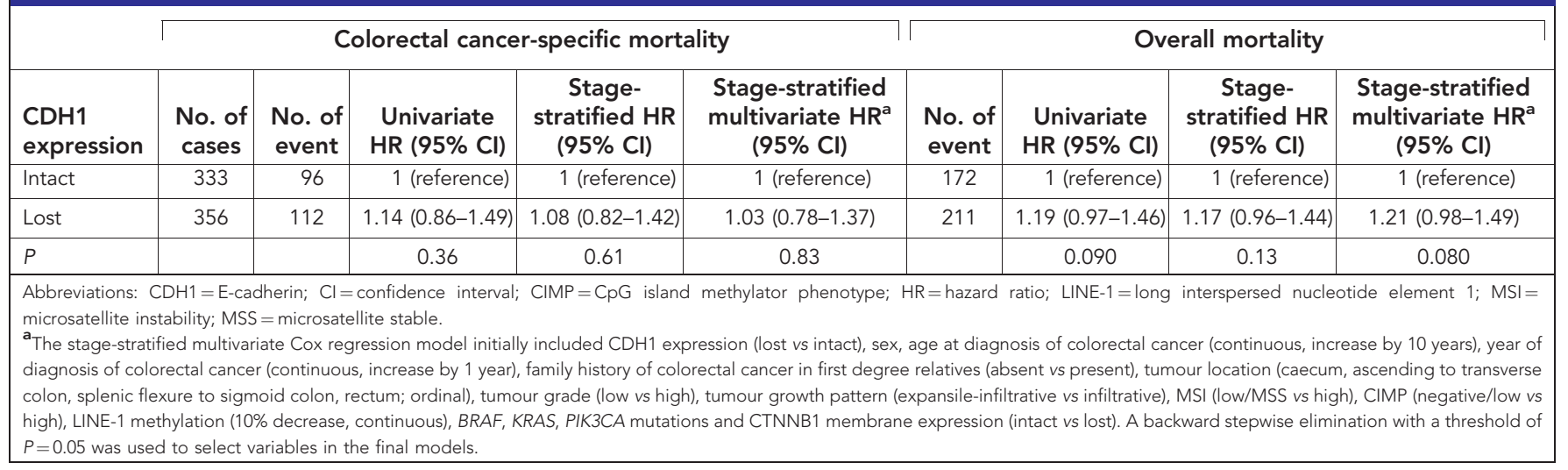


growth pattern-associated molecular marker has examined as many molecular variables as we did in this study.

This study has limitations. First, we used TMA to assess the tumour $\mathrm{CDH} 1$ expression. Although a previous study utilising TMA for CDH1 expression in colorectal cancer showed that there was no significant difference in the distribution of $\mathrm{CDH} 1$ between tumour centre and invasive front (Kroepil et al, 2013), we must recognise the limitation of TMA-based assessment. A subset of tumours with partial or heterogeneous positivity in whole sections might have been scored as 'negative' in TMA cores. This potential misclassification of tumours in terms of CDH1 expression would be expected to be unrelated to tumour growth pattern, and hence would have driven our results towards the null hypothesis. Despite this limitation, we were able to demonstrate the significant and independent relation of loss of CDH1 expression with the infiltrative tumour growth pattern and $\mathrm{pN}$ stage. Second, data on treatment after the diagnosis of cancer were limited. However, it is unlikely that the distribution of chemotherapy use could substantially differ according to tumour $\mathrm{CDH} 1$ expression, because the data on tumour $\mathrm{CDH} 1$ expression were not available for treatment decisions. Third, data on cancer recurrence were not available. Nonetheless, with long follow-up period of those who were censored, colorectal cancer-specific mortality is a reasonable surrogate of colorectal cancer-specific outcome endpoint.

In conclusion, this study provides evidence for the association of tumour $\mathrm{CDH} 1$ expression with infiltrative tumour growth pattern and lymph node metastasis independent of clinical, pathological and tumour molecular features in colorectal cancer.

\section{ACKNOWLEDGEMENTS}

This work was supported by U.S. National Institutes of Health (NIH) grants (K07 CA190673 to RN; R01 CA137178 and K24 DK098311 to ATC; P50 CA127003 to CSF; R01 CA151993 and R35 CA197735 to SO; P01 CA87969 and UM1 CA186107 to Meir J Stampfer, Nurse's Health Study; P01 CA55075 and UM1 CA167552 to Walter C Willett, Health Professional's Follow-up Study); grants from the Paula and Russell Agrusa Fund for Colorectal Cancer Research, the Friends of the Dana-Farber Cancer Institute, the Bennett Family Fund and the Entertainment Industry Foundation through National Colorectal Cancer Research Alliance. SAK was supported by an early exchange postdoctoral fellowship grant from Asan Medical Center, Korea. KI was supported by a Japan Society for the Promotion of Science Postdoctoral Fellowship for Research Abroad and by Takashi Tsuruo Memorial Fund, Japan. KM was supported by a fellowship grant from the Uehara Memorial Foundation, Japan. ATC is a Damon Runyon Clinical Investigator. We would like to thank the participants and staff of the Nurses' Health Study and the Health Professionals Follow-up Study for their valuable contributions, as well as the following state cancer registries for their help: AL, AZ, AR, CA, CO, CT, DE, FL, GA, ID, IL, IN, IA, KY, LA, ME, MD, MA, MI, NE, NH, NJ, NY, NC, ND, OH, OK, OR, PA, RI, SC, TN, TX, VA, WA, WY. In addition, this study was approved by the Connecticut Department of Public Health (DPH) Human Investigations Committee. Use of standardised official symbols: We use HUGO (Human Genome Organisation)-approved official symbols for genes and gene products, including $B R A F$; CDH1 (so-called E-cadherin); CTNNB1 (catenin (cadherin-associated protein), beta $1,88 \mathrm{kDa}$; so-called $\beta$-catenin); KRAS; and PIK3CA; all of which are described at www.genenames.org.

\section{CONFLICT OF INTEREST}

ATC previously served as a consultant for Bayer Healthcare, Millennium Pharmaceuticals, Pozen Inc and Pfizer Inc. This study was not funded by Bayer Healthcare, Millennium Pharmaceuticals, Pozen Inc, or Pfizer Inc. The remaining authors declare no conflicts of interest.

\section{DISCLAIMER}

Certain data used in this publication were obtained from the DPH. The authors assume full responsibility for analyses and interpretation of these data.

\section{REFERENCES}

Bondi J, Bukholm G, Nesland JM, Bakka A, Bukholm IR (2006) An increase in the number of adhesion proteins with altered expression is associated with an increased risk of cancer death for colon carcinoma patients. Int J Colorectal Dis 21(3): 231-237.

Chen X, Wang Y, Xia H, Wang Q, Jiang X, Lin Z, Ma Y, Yang Y, Hu M (2012) Loss of E-cadherin promotes the growth, invasion and drug resistance of colorectal cancer cells and is associated with liver metastasis. Mol Biol Rep 39(6): 6707-6714.

Cianchi F, Messerini L, Comin CE, Boddi V, Perna F, Perigli G, Cortesini C (2007) Pathologic determinants of survival after resection of T3N0 (Stage IIA) colorectal cancer: proposal for a new prognostic model. Dis Colon Rectum 50(9): 1332-1341.

Fadare O, Reddy H, Wang J, Hileeto D, Schwartz PE, Zheng W (2005) E-Cadherin and beta-Catenin expression in early stage cervical carcinoma: a tissue microarray study of 147 cases. World J Surg Oncol 3: 38.

Filiz AI, Senol Z, Sucullu I, Kurt Y, Demirbas S, Akin ML (2010) The survival effect of E-cadherin and catenins in colorectal carcinomas. Colorectal Dis 12(12): 1223-1230.

He X, Chen Z, Jia M, Zhao X (2013) Downregulated E-cadherin expression indicates worse prognosis in Asian patients with colorectal cancer: evidence from meta-analysis. PLoS One 8(7): e70858.

Hinoue T, Weisenberger DJ, Lange CP, Shen H, Byun HM, Van Den Berg D, Malik S, Pan F, Noushmehr H, van Dijk CM, Tollenaar RA, Laird PW (2012) Genome-scale analysis of aberrant DNA methylation in colorectal cancer. Genome Res 22(2): 271-282.

Imamura Y, Lochhead P, Yamauchi M, Kuchiba A, Qian ZR, Liao X, Nishihara R, Jung S, Wu K, Nosho K, Wang YE, Peng S, Bass AJ, Haigis KM, Meyerhardt JA, Chan AT, Fuchs CS, Ogino S (2014) Analyses of clinicopathological, molecular, and prognostic associations of KRAS codon 61 and codon 146 mutations in colorectal cancer: cohort study and literature review. Mol Cancer 13: 135.

Irahara N, Baba Y, Nosho K, Shima K, Yan L, Dias-Santagata D, Iafrate AJ, Fuchs CS, Haigis KM, Ogino S (2010) NRAS mutations are rare in colorectal cancer. Diagn Mol Pathol 19(3): 157-163.

Jie D, Zhongmin Z, Guoqing L, Sheng L, Yi Z, Jing W, Liang Z (2013) Positive expression of LSD1 and negative expression of E-cadherin correlate with metastasis and poor prognosis of colon cancer. Dig Dis Sci 58(6): 1581-1589.

Jonker DJ, Karapetis CS, Harbison C, O’Callaghan CJ, Tu D, Simes RJ, Malone DP, Langer C, Tebbutt N, Price TJ, Shapiro J, Siu LL, Wong RP, Bjarnason G, Moore MJ, Zalcberg JR, Khambata-Ford S (2014) Epiregulin gene expression as a biomarker of benefit from cetuximab in the treatment of advanced colorectal cancer. Br J Cancer 110(3): 648-655.

Karamitopoulou E, Zlobec I, Koelzer VH, Langer R, Dawson H, Lugli A (2015) Tumour border configuration in colorectal cancer: proposal for an alternative scoring system based on the percentage of infiltrating margin. Histopathology 67(4): 464-473.

Karamitopoulou E, Zlobec I, Patsouris E, Peros G, Lugli A (2011) Loss of E-cadherin independently predicts the lymph node status in colorectal cancer. Pathology 43(2): 133-137.

Keum M, Lim S-B, Kim SA, Yoon YS, Kim CW, Yu CS, Kim JC (2012) Clinicopathologic factors affecting recurrence after curative surgery for stage I colorectal cancer. J Korean Soc Coloproctol 28(1): 49-55.

Kroepil F, Fluegen G, Vallbohmer D, Baldus SE, Dizdar L, Raffel AM, Hafner D, Stoecklein NH, Knoefel WT (2013) Snaill expression in colorectal cancer and its correlation with clinical and pathological parameters. BMC Cancer 13: 145. 
Kwak JM, Min BW, Lee JH, Choi JS, Lee SI, Park SS, Kim J, Um JW, Kim SH, Moon HY (2007) The prognostic significance of E-cadherin and liver intestine-cadherin expression in colorectal cancer. Dis Colon Rectum 50(11): 1873-1880.

Liao X, Lochhead P, Nishihara R, Morikawa T, Kuchiba A, Yamauchi M, Imamura Y, Qian ZR, Baba Y, Shima K, Sun R, Nosho K, Meyerhardt JA, Giovannucci E, Fuchs CS, Chan AT, Ogino S (2012) Aspirin use, tumor PIK3CA mutation, and colorectal-cancer survival. N Engl J Med 367(17): 1596-1606.

Lochhead P, Kuchiba A, Imamura Y, Liao X, Yamauchi M, Nishihara R, Qian ZR, Morikawa T, Shen J, Meyerhardt JA, Fuchs CS, Ogino S (2013) Microsatellite instability and BRAF mutation testing in colorectal cancer prognostication. J Natl Cancer Inst 105(15): 1151-1156.

Lu MH, Huang CC, Pan MR, Chen HH, Hung WC (2012) Prospero homeobox 1 promotes epithelial-mesenchymal transition in colon cancer cells by inhibiting E-cadherin via miR-9. Clin Cancer Res 18(23): 6416-6425.

Lugli A, Zlobec I, Minoo P, Baker K, Tornillo L, Terracciano L, Jass JR (2007) Prognostic significance of the wnt signalling pathway molecules APC, beta-catenin and E-cadherin in colorectal cancer: a tissue microarraybased analysis. Histopathology 50(4): 453-464.

Morikawa T, Kuchiba A, Qian ZR, Mino-Kenudson M, Hornick JL, Yamauchi M, Imamura Y, Liao X, Nishihara R, Meyerhardt JA, Fuchs CS, Ogino S (2012) Prognostic significance and molecular associations of tumor growth pattern in colorectal cancer. Ann Surg Oncol 19(6): 1944-1953.

Morikawa T, Kuchiba A, Yamauchi M, Meyerhardt JA, Shima K, Nosho K, Chan AT, Giovannucci E, Fuchs CS, Ogino S (2011) Association of CTNNB1 (beta-catenin) alterations, body mass index, and physical activity with survival in patients with colorectal cancer. JAMA 305(16): 1685-1694.

Nosho K, Irahara N, Shima K, Kure S, Kirkner GJ, Schernhammer ES, Hazra A, Hunter DJ, Quackenbush J, Spiegelman D, Giovannucci EL, Fuchs CS, Ogino S (2008) Comprehensive biostatistical analysis of CpG island methylator phenotype in colorectal cancer using a large populationbased sample. PLoS One 3(11): e3698.

Ogino S, Brahmandam M, Cantor M, Namgyal C, Kawasaki T, Kirkner G, Meyerhardt JA, Loda M, Fuchs CS (2006a) Distinct molecular features of colorectal carcinoma with signet ring cell component and colorectal carcinoma with mucinous component. Mod Pathol 19(1): 59-68.

Ogino S, Brahmandam M, Kawasaki T, Kirkner GJ, Loda M, Fuchs CS (2006b) Combined analysis of COX-2 and p53 expressions reveals synergistic inverse correlations with microsatellite instability and $\mathrm{CpG}$ island methylator phenotype in colorectal cancer. Neoplasia 8(6): 458-464.

Ogino S, Kawasaki T, Brahmandam M, Cantor M, Kirkner GJ, Spiegelman D, Makrigiorgos GM, Weisenberger DJ, Laird PW, Loda M, Fuchs CS (2006c) Precision and performance characteristics of bisulfite conversion and real-time PCR (MethyLight) for quantitative DNA methylation analysis. J Mol Diagn 8(2): 209-217.

Ogino S, Kawasaki T, Brahmandam M, Yan L, Cantor M, Namgyal C, Mino-Kenudson M, Lauwers GY, Loda M, Fuchs CS (2005a) Sensitive sequencing method for KRAS mutation detection by Pyrosequencing. J Mol Diagn 7(3): 413-421.
Ogino S, Kawasaki T, Kirkner GJ, Kraft P, Loda M, Fuchs CS (2007) Evaluation of markers for $\mathrm{CpG}$ island methylator phenotype (CIMP) in colorectal cancer by a large population-based sample. J Mol Diagn 9(3): 305-314.

Ogino S, Kawasaki T, Nosho K, Ohnishi M, Suemoto Y, Kirkner GJ, Fuchs CS (2008) LINE-1 hypomethylation is inversely associated with microsatellite instability and $\mathrm{CpG}$ island methylator phenotype in colorectal cancer. Int J Cancer 122(12): 2767-2773.

Ogino S, Meyerhardt JA, Cantor M, Brahmandam M, Clark JW, Namgyal C, Kawasaki T, Kinsella K, Michelini AL, Enzinger PC, Kulke MH, Ryan DP, Loda M, Fuchs CS (2005b) Molecular alterations in tumors and response to combination chemotherapy with gefitinib for advanced colorectal cancer. Clin Cancer Res 11(18): 6650-6656.

Ogino S, Nosho K, Kirkner GJ, Kawasaki T, Meyerhardt JA, Loda M, Giovannucci EL, Fuchs CS (2009) CpG island methylator phenotype, microsatellite instability, BRAF mutation and clinical outcome in colon cancer. Gut 58(1): 90-96.

Qian ZR, Sano T, Yoshimoto K, Asa SL, Yamada S, Mizusawa N, Kudo E (2007) Tumor-specific downregulation and methylation of the CDH13 (H-cadherin) and $\mathrm{CDH} 1$ (E-cadherin) genes correlate with aggressiveness of human pituitary adenomas. Modern Pathol 20(12): 1269-1277.

Roman R, Verdu M, Calvo M, Vidal A, Sanjuan X, Jimeno M, Salas A, Autonell J, Trias I, Gonzalez M, Garcia B, Rodon N, Puig X (2010) Microsatellite instability of the colorectal carcinoma can be predicted in the conventional pathologic examination. A prospective multicentric study and the statistical analysis of 615 cases consolidate our previously proposed logistic regression model. Virchows Arch 456(5): 533-541.

Toth L, Andras C, Molnar C, Tanyi M, Csiki Z, Molnar P, Szanto J (2012) Investigation of beta-catenin and E-cadherin expression in Dukes B2 stage colorectal cancer with tissue microarray method. Is it a marker of metastatic potential in rectal cancer? Pathol Oncol Res 18(2): 429-437.

Tsanou E, Peschos D, Batistatou A, Charalabopoulos A, Charalabopoulos K (2008) The E-cadherin adhesion molecule and colorectal cancer. A global literature approach. Anticancer Res 28(6A): 3815-3826.

Zlobec I, Baker K, Minoo P, Hayashi S, Terracciano L, Lugli A (2009) Tumor border configuration added to TNM staging better stratifies stage II colorectal cancer patients into prognostic subgroups. Cancer 115(17): 4021-4029.

Zlobec I, Hadrich M, Dawson H, Koelzer VH, Borner M, Mallaev M, Schnuriger B, Inderbitzin D, Lugli A (2014) Intratumoural budding (ITB) in preoperative biopsies predicts the presence of lymph node and distant metastases in colon and rectal cancer patients. Br J Cancer 110(4): 1008-1013.

Zlobec I, Lugli A, Baker K, Roth S, Minoo P, Hayashi S, Terracciano L, Jass JR (2007) Role of APAF-1, E-cadherin and peritumoral lymphocytic infiltration in tumour budding in colorectal cancer. J Pathol 212(3): 260-268.

This work is published under the standard license to publish agreement. After 12 months the work will become freely available and the license terms will switch to a Creative Commons AttributionNonCommercial-Share Alike 4.0 Unported License.

Supplementary Information accompanies this paper on British Journal of Cancer website (http://www.nature.com/bjc) 